\title{
Study on fatigue load of vehicle body based on line test
}

\author{
Wang Ziyuan \\ Traction Power State Key Laboratory ,Southwest Jiao Tong University,Chengdu 610031 ,China \\ sanluke@qq.com
}

Key words: Dynamic load, Fatigue, Acceleration, Strain, Line test

\begin{abstract}
In this paper, it's based on the acceleration and strain data from the test of a city railway vehicle .This paper introduced how to carry out the excitation from the center pivot, air spring and couple by numerical integration and finite element model and get the external load of the car body.
\end{abstract}

\section{Introduction}

Nowadays, the city railway traffic is developing. but when the speed is meeting the social requirements, there are also some problems comes. Vehicle parts, especially the body structure which is the main bearing structure, suffers from track excitation from the bogie ,the longitudinal excitation from the couple, and the traction excitation for EMU bogie series.The alternating excitation of these is worked on the car body at the same time. It's a challenge for the car body fatigue strength in such a complex working enviroment. In order to improve the stability and reliability of the operation of the train , strength evaluation is particularly important. In this paper, the load condition which is based on the real line experiment of the vehicle for the fatigue evaluation is studied and optimized.

\section{Sensor arrangement}

There are two main types of sensors, one is acceleration sensor, mainly measure the acceleration of vibration, is usually used to measure the transmission of excitation and vibration.

The other One is the strain sensor, which is used to measure the strain, and the stress is calculated by the strain.

The external load of the vehicle mainly directly come from the shock absorber in the secondary suspension, the traction devices, and the couple.

\subsection{The sensor arrangement of center pivot}

The longitudinal and lateral loads is mainly caused by the train pulling force.We chose the stain sensor to get the stress of the center pivot,and we can see the arrangement of the sensor below in Fig[1].The reason of the arrangement will be explained in the paper after.

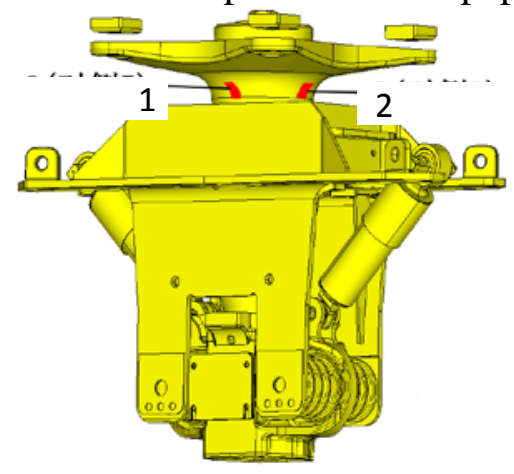

Fig.1 The sensor position of center pivot

\subsection{The sensor arrangement of secondary suspension}

The body parts (bolster beam) and the bogie frame which is under the air bag is where we set the acceleration sensors to measure the vibration acceleration of the car body and the bogie frame. The arrangement is based on the need of establishing the dynamic model of the air bag .Our set is as the 
Fig[2] below:

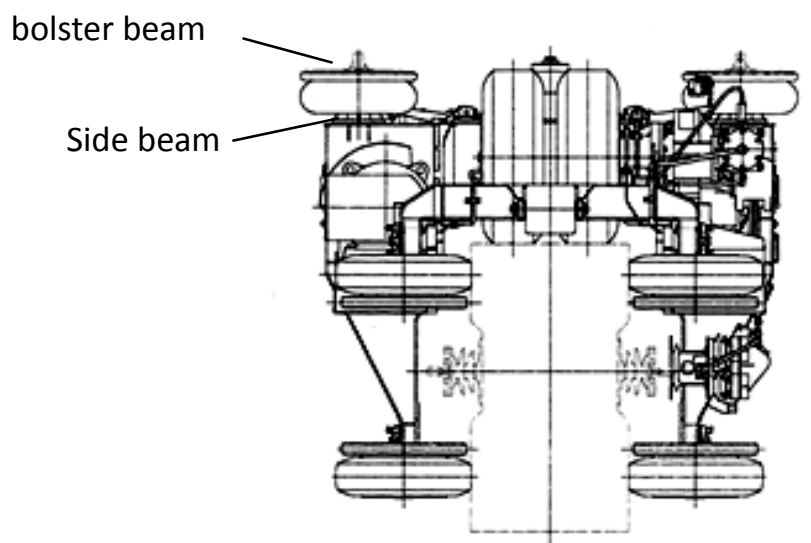

Fig.2 The sensor position of secondary suspension

\subsection{The sensor arrangement of coupler}

We set the strain sensor on the coupler along track direction.As the coupler is special ,it has a part which is rigid connected to the car body without any damper inside,and we set the sensor in this part for an easier calculation.So we can measure the coupler longitudinal stress and inversion body longitudinal load.The arrangement is as shown in Fig [3]:

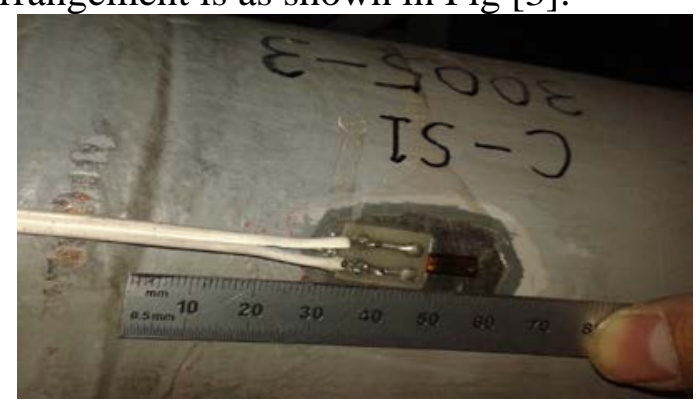

Fig.3 The sensor position of coupler

\section{The longitudinal and lateral loads from the traction transfer device}

By using the strain sensor data, we can calculate stress data. These loads passing from the moto to the car body through the center pivot can be inverted by the dynamic stress. The process can be inverted by the finite element software. This paper introduces the software FEM inversion, the software we use here is abaqus.

The finite element model of the center pivot is established, and the stress distribution is calculated by using the C3D10M element, which is shown in Fig[4]:

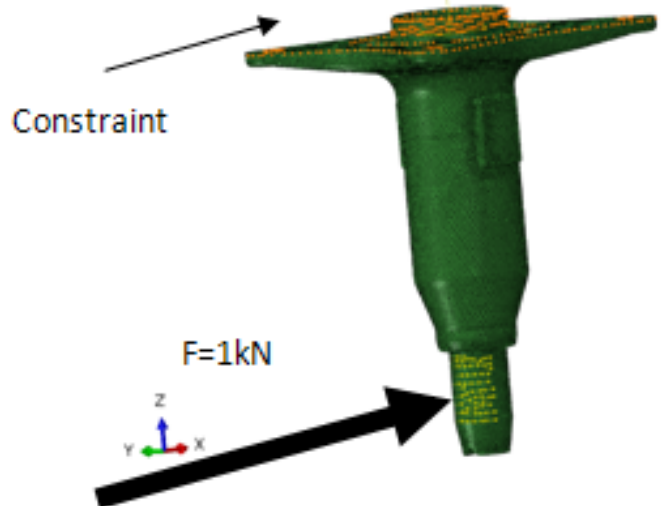

Fig.4 Finite element model

Constraint:The up surface of the center pivot is restricted by Z-Freedom and the four bolts holes is restricted by $\mathrm{X}$-Freedom and Y-Freedom.

By applying the force of longitudinal $1 \mathrm{kN}$, the response factor of the stress of two points is $\alpha_{1}$, $\alpha_{2}$.It is also need for local coordinate transformation, as shown in Fig [5]: 


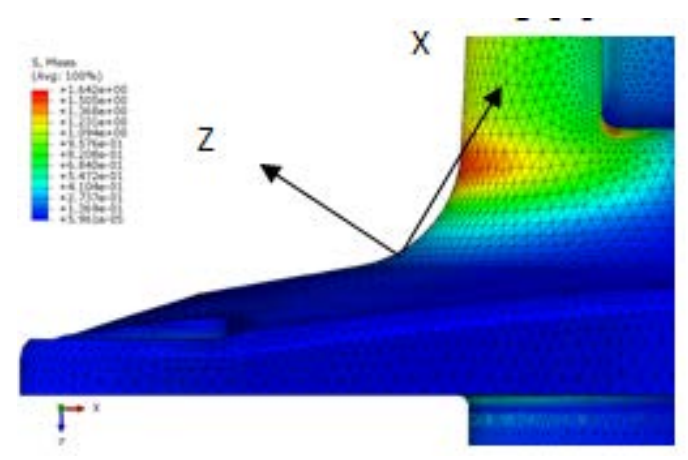

Fig.5 Coordinate transformation

We also set the force of lateral $1 \mathrm{kN}$, the response factor of the lateral force to the stress of the two points is $\beta_{1}, \beta_{2}$.

Response equation can be obtained:

$$
\left\{\begin{array}{l}
\alpha_{1} X+\beta_{1} Y=\sigma_{1} \\
\alpha_{2} X+\beta_{2} Y=\sigma_{2}
\end{array}\right.
$$

$\alpha_{1}$ : The response factor of the longitudinal stress at the position of the strain sensor 1

$\alpha_{2}$ :The response factor of the longitudinal stress at the position of the strain sensor 2

$\beta_{1}$ :The response factor of the lateral stress at the position of the strain sensor 1

$\beta_{2}$ :The response factor of the lateral stress at the position of the strain sensor 2

$\mathrm{X}$ : The longitudinal load

$\mathrm{Y}$ : The lateral load

$\sigma_{1}$ : The test data of the strain sensor 1

$\sigma_{2}$ : The test data of the strain sensor 2

By solving the equation, we can get the longitudinal force and lateral force. And by the test stress time series, we can get the longitudinal force and lateral force load load time series which can be used to evaluate the fatigue.

\section{Longitudinal load from the coupler}

The load from the coupler in the structural response can be inverted by unit load strain,this paper assume that the section of the coupler is a ring,and so we can get the response factor $\lambda$ by coupler outer diameter $\mathrm{D}$, inner diameter $\mathrm{d}$.

$$
\lambda=\frac{F}{S}=\frac{F}{\pi \cdot\left(D^{2}-d^{2}\right)}
$$

The measured stress time series multiplied by the response factor, load time series can be obtained by the coupler longitudinal force.

\section{The vertical load from the secondary suspension}

In this paper, a new method is presented for the inversion of the vertical load of the vehicle which uses the air spring as the secondary suspension, which is more complex and requires consideration of the damping effect.

Because of its excellent accuracy and frequency response , acceleration sensor is widely used in the vibration experiment.Comparing with the displacement sensor, the acceleration sensor has less interference, higher sensitivity, higher sampling frequency. So we use the acceleration sensor data for our inversion.

First of all, we need to establish the dynamic model of the spring [1], Fig[6] shows the general air bag dynamic model, in which the $\mathrm{Zp}$ is the intermediate mass coordinates, $\mathrm{K}_{0}$ is the air stiffness, $\mathrm{K}_{1}$ is the air bag stiffness, $\mathrm{c} *$ is the air spring damping. 


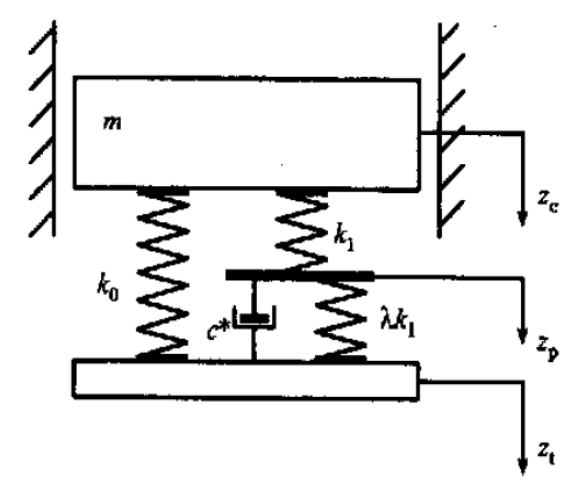

Fig.6 Air bag dynamic model

We can obtain the following equation by using the model.

$$
\begin{gathered}
\mathrm{m} \frac{\mathrm{d}^{2} z_{c}}{d t^{2}}=\mathrm{k}_{0} \mathrm{z}_{\mathrm{t}}+\mathrm{k}_{1} \mathrm{z}_{\mathrm{p}}-\left(\mathrm{k}_{0}+\mathrm{k}_{1}\right) \mathrm{z}_{\mathrm{c}} \\
c^{*} \frac{d z_{p}}{d t}+(1+\lambda) k_{1} z_{P}-k_{1} \mathrm{z}_{c}=c^{*} \frac{d \mathrm{z}_{t}}{d t}+\lambda k_{1} \mathrm{z}_{t}
\end{gathered}
$$

$\mathrm{Zt}$, Zc can be obtained by the experimental measurement of the acceleration load (integrated two times,but we must ensure that the integral initial constant is 0 ), the air stiffness $\mathrm{K}_{0}$, air bag stiffness $\mathrm{K}_{1}$ and air spring damping $\mathrm{c}^{*}$ can be determined by the air bag's properties. $\lambda$ is the ratio of the air bag working volume and the auxiliary bag volume, this paper assume the condition of the maximum load.

Due to the equivalent mass $\mathrm{M}$, it is not sure to be determined in the dynamic process. Therefore, we need to find the force of the air bag passing to the vehicle body.

So the value of $\mathrm{Zp}$ is our main problems need to be solved, because our experimental data is a random data, and cannot get accurate numerical solutions.In order to get the differential solution in the secondary equation, this paper choose $4^{\text {th }}$-order Runge-Kutta method [2].

Runge-Kutta method is a kind of single step method with high accuracy in engineering, which is used to solve the differential equations. The numerical solution is as follows:

$$
\left\{\begin{array}{l}
\mathrm{Y}(\mathrm{i}+1)=\mathrm{y}(\mathrm{I})+\mathrm{h} *(2 * \mathrm{~K} 2+2 * \mathrm{~K} 3+\mathrm{K} 4 \mathrm{~K} 1+) / 6 \\
\mathrm{~K} 1=\mathrm{f}(\mathrm{x}(\mathrm{I}), \mathrm{y}(\mathrm{I})) \\
\mathrm{K} 2=\mathrm{f}(\mathrm{x}(\mathrm{I})+\mathrm{h} / 2, \mathrm{y}(\mathrm{I})+\mathrm{h} * \mathrm{~K} 1 / 2) \\
\mathrm{K} 3=\mathrm{f}(\mathrm{x}(\mathrm{I})+\mathrm{h} / 2, \mathrm{y}(\mathrm{I})+\mathrm{h} * \mathrm{~K} 2 / 2) \\
\mathrm{K} 4=\mathrm{f}(\mathrm{x}(\mathrm{I})+\mathrm{h}, \mathrm{y}(\mathrm{I})+\mathrm{h} * \mathrm{~K} 3)
\end{array}\right.
$$

$\mathrm{K} 1$ : the slope at the beginning time;

$\mathrm{K} 2$ : the slope of time point, the Euler slope $\mathrm{K} 1$ to determine $\mathrm{y}$ in $\mathrm{T}_{\mathrm{N}}+\mathrm{h} / 2$ value;

$\mathrm{K} 3$ : also the midpoint of the slope, but this time using the slope of $\mathrm{K} 2$ to determine the $\mathrm{y}$ value;

$\mathrm{K} 4$ : the slope at the end of time, and its y value is determined by K3;

$\mathrm{h}$ : the unit step, according to the data of the sampling frequency of $1000 \mathrm{~Hz}$, the step size is 0.001 Because the initial condition of the load displacement is 0 , the $X(0)=0, Y(0)=0$.

With the experiment data by using MATLAB, we can calculate the approximation of intermediate mass coordinates $\mathrm{Zp}$, and take it into the first equation we can obtain the air spring vertical force.(we should notice that force whether to add the $1 / 4$ body quality into calculation depends on the vehicle loading model .If the inertia relief method is used to computes the quasi-static factor [3] in fatigue calculation ,there's no need to combine with body mass.)

\section{Postprocessing of load data and fatigue evaluation method}

The car body is not sensitive to the high frequency vibration response (for local mode, the finite 
element evaluation should be considered particularly ).We must filter the load signal by bandpassing to filter the high frequency signal which make little contribution to the real strain of the car body and the very low frequency signal which may caused by the error of the sensor.

Then we calculate the quasi-static factors in finite element software, and the method is similar to the inversion of the load from the center pivot, but the stress is used as the input of the load spectrum, then the stress spectrum of the load spectrum can be obtained.Then we can use fatigue software to like nCode to evaluate the fatigue.

\section{Summary}

The body fatigue state based on experimental data can accurately predict the vehicle condition especially for the vehicle which running on the confirmed line like the city rail transit vehicles.And rely on the system, we can not only calculate the body fatigue, but also establish the strength mining system by series of easy calculation.It can be directly get through a small amount of the sensor observation.Vehicle dynamic stress change ,especially the damage, can be detected,so we can repair the vehicle in time.But this method is still dependent on quasi-static calculation, and can not really calculate the transient response.It is suggested we could take in consideration of vehicle model to approach the actual transient response of the vehicle . But the data we get is also the response data, it may be affected by the feedback signal of the car body and the car body model may change as the result of the passenger and interior decoration. How to fix these is a further problem we should work on.

\section{References}

[1]Li Fu,Fu Maohai,Huang Yunhu,Ni Wenbo,The study on the dynamic model of air bag,J.China Railway Science,24(2003)91-95.

[2]Zhao Qiuling,The accuracy method of nonlinear dynamic equation,J.Mechanic and Experiment,6(1998)34-36.

[3]Liao Bingrong,Zhang Weihua,Jing Dingsheng,Xiao Shoune,The dynamic strain of car body structure,J.Journal of Traffic and Transportation Engineering,7(2007)18-20.

[4]Kenjiro Goda, Takaomi Nishigaito. A Curving Simulation for a Monorail Car,in: Railroad Conference,Proceedings of the 2000 ASME/IEEE Joint,2000.171-177.

[5]R.Schwertasek,R.E.Roberson.Dynamic of Multibody System.Berlin:Springer- Verlag(1986)

[6]W.Kortum. Review of Multibody Computer Codes for Vehicle System Dynamics. Vehicle System Dynamics(1993)

[7]Kadhim,R.J.,Vlahakavis,P.Speeifieation of rolling stock power capacity,in:IEE Conferenece Publieation,453(1998)14-17. 